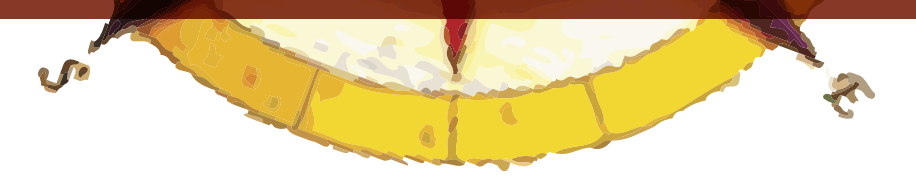

\title{
UNA EXPERIENCIA EDUCATIVA SOBRE CIUDADANÍA: CURSO B LEARNING FORMACIÓN PARA LA DEMOCRACIA Y EL EJERCICIO DE LOS DERECHOS
}

\author{
Marisol Campillay Llanos ${ }^{20}$ \\ Nataly Pérez Cisternas ${ }^{21}$
}

\section{RESUMEN}

Este artículo presenta algunos aspectos sobre los resultados obtenidos al termino del curso b-learning Formación para la democracia y el ejercicio de los derechos, el cual fue la segunda iniciativa en el área de ciencias sociales desarrollada por el equipo del Programa de Investigaciones e Intervenciones Territoriales-PIIT de la Universidad Academia de Humanismo Cristiano-UAHC en asociación con el Ministerio de Educación de Chile-MINEDUC y el Centro de perfeccionamiento e investigaciones pedagógicas -CPEIP.

Este curso fue una apuesta por re-leer el curriculum escolar, especialmente en el sector de Historia, Geografía y Ciencias Sociales, privilegiando el desarrollo de una pedagogía praxiológica, donde los docentes motoricen procesos de enseñanza y aprendizaje, que permitan la comprensión y la reelaboración social del pasado a las nuevas generaciones, para participar activamente de su presente.

Palabras clave: educación ciudadana, curriculum escolar.

\section{RESUMO}

Este artigo apresenta alguns aspectos sobre os resultados obtidos na conclusão do curso b-learning Formación para la democracia y el ejercicio de los derechos, sendo esta a segunda iniciativa na área das ciências sociais desenvolvida pela equipe do Programa de Investigaciones e Intervenciones Territoriales-PIIT da Universidade Academia de Humanismo Cristiano-UAHC em associação com o Ministerio de Educación de Chile-MINEDUC e o Centro de perfeccionamiento e investigaciones pedagógicas -CPEIP.

Este curso foi uma aposta por re-ler o curriculo escolar, especialmente no setor História, Geografia e Ciências Sociais, privilegiando o desenvolvimento duma pedagogia praxiológica, onde os docentes desenvolvam processos de ensino e aprendizagem, que permitam a compreensão e reelaboração do passado das novas gerações, para participar ativamente de seu presente.

20 Candidata a Magíster en Educación Pontificia Universidad Católica de Chile.

21 Candidata a Magíster en Geografía Universidad Federal de Goiás. Brasil. 
Palavras chave: educação cidadania, curriculo escolar.

\begin{abstract}
This article presents some aspects of the results obtained on completion of the course b-learning Formación para la Democracia y el ejercicio de los derechos, which is the second initiative in the social sciences developed by the Programa de Intervenciones y Investigaciones Territoriales - PIIT staff of Universidad Academia de Humanismo Cristiano UAHC - in association with the Ministry of Education of Chile-MINEDUCand the Centro deperfeccionamientoeinvestigacionespedagógicas-CPEIP. This course is a bet for re-reading the school curriculum, especially in the History, Geography and Social Sciences, focusing on the development of a praxeological pedagogy, where teachers develop teaching and learning process, allowing the past understanding and reworking of the new generations, to participate actively in the present.
\end{abstract}

Keywords: citizenship education, school curriculum.

\title{
ALGUNAS PALABRAS PARA COMENZAR
}

Esta iniciativa surge en el marco de la actualización del currículum chileno (2009), que proviene de la propuesta establecida durante los gobiernos de la concertación, esta actualización apuntó al fortalecimiento de las herramientas conceptuales, actitudinales y procedimentales que los profesores de enseñanza básica y enseñanza media ${ }^{22}$, debiesen desarrollar para producir cambios y transformaciones en sus prácticas educativas y cotidianas.

En este sentido, el curso Formación para la democracia y el ejercicio de los derechos se enfoco en la enseñanza y prácticas de la ciudadanía considerando por un lado, la necesidad del profesor por actualizarse y profundizar rigurosamente sus conocimientos disciplinares, sobre la historia reciente de Chile y la humanidad, con el objetivo de promover la discusión de distintos procesos históricos, sus contextos y las variadas explicaciones y comprensiones que se levantan sobre los mismos, y por otro lado, pensar en las múltiples posibilidades que el currículum ofrece para reflexionar sobre el tema, desde una visión integradora y transversal y no sólo como actos aislados o actividades extraprogramáticas, destacando que la educación en ciudadanía, tiene deberes y obligaciones éticas, lo que obliga a una transformación de las relaciones sociales en el aula y sus modelos didácticos, para permitir que la deliberación y la opinión del otro, se instale en el espacio escolar, bajo patrones de respeto, pero evitando cualquier relativización ética.

\section{TENSIONES PRE-EXISTENTES: ACTUALIZACIÓN CURRICULAR UN CONFLICTO LATENTE}

Los fundamentos que se presentan en el marco curricular (2009) tienen su origen en la reforma curricular de los 90', la cual se sustenta en decretos que permiten que el curriculum nacional sea

22 Considerando que esta actualización, hoy solo es efectiva hasta primer año medio en términos concretos, quedando segundo, tercero y cuarto año medio a la espera de cambios permanentes, ya que este proceso fue pensado en etapas, sin embargo, y por causa del cambio de gobierno, que significo el retorno de los gobiernos de derecha a la dirección del país, este proceso se encuentra en reestructuración. 
obligatorio para todas las escuelas del país, sin embargo se sitúa sobre un escenario dinámico que permite que este sea sometido a diversas actualizaciones, solventando las demandas que presenta la sociedad. Las innovaciones desarrolladas en el 2009, surgen también producto de dar solución a algunas problemáticas que se han presentado en la implementación curricular, respondiendo a la propuesta del escenario dinámico, en que se debe configurar el curriculum "se presenta un diagnóstico del currículum de la Reforma analizando sus fortalezas y debilidades, y dando cuenta de las críticas y demandas que distintos actores del sistema educativo han ido levantando tras una década de implementación" (Ministerios de educación, 2009, p. 1). En este sentido, es que se construye la base en la que se instalo el curso Formación para la democracia y el ejercicio de los derechos y por supuesto, el profesorado que forma parte del proceso de implementación curricular.

El autor Escudero (1988) manifiesta que la innovación curricular responde a "Un proceso de definición, construcción y participación social" (p.86). En primera instancia es preciso ahondar el lugar que ocupa el docente en los ajustes curriculares 2009, como también los aportes que brinda el curso Formación para la democracia y el ejercicio de los derechos a la actualización 2009. El profesorado en el periodo de propuesta de ajustes curriculares argumenta: "La forma de imponer estos ajustes no corresponde al carácter democrático que una sociedad debe tener. No fueron consultados los profesores, no fueron consultados los organismos gremiales, no fueron consultados los especialistas (...)" (Colegio de profesores, 2010)

La cita clarifica que las actualizaciones curriculares planteadas por el Ministerio de Educación (2009) no responden a una lógica de consenso atendiendo a la comunidad educativa que va a ser parte principal en el proceso de implementación, de esta forma no se identificarían los presupuestos de innovación curricular como lo propone Escudero (1988), evidenciándose una falta de espacio de participación social.

Frente a estas tensiones y malestares surge el curso Formación para la democracia y el ejercicio de los derechos, considerando que no sólo el contexto político-social se encontraba desestabilizado debido a los resquemores que presupone una actualización del curriculum, sino que también debido a los cambios en la escena política del país que dejaba su etapa concertacionista de centro-izquierda para situarse, por medio de la vía democrática, en un nuevo período de derecha que sacaba a flote la memoria colectiva sobre los derechos humanos y la ciudadanía, es así como sin presuponerlo la formación ciudadana paso a ocupar un lugar privilegiado en el debate nacional.

\section{¿QUÉ QUEREMOS DECIR, CUANDO HABLAMOS DE FORMACIÓN CIUDADANA?}

Al comenzar a hablar de ciudadanía, las definiciones parecen ser múltiples, sin embargo coinciden en aspectos primordiales, tal cual José Gimeno Sacristán (2001) lo señala:

Es una forma de ser persona en sociedad que parte del reconocimiento del individuo como poseedor de unas posibilidades y de unos derechos. Se trata de una construcción históricamente muy elaborada, cuya esencia radica en comprendernos y respetarnos como libres, autónomos e iguales, al tiempo que se vive con otros; condición de la que se deriva una forma de percibirse a sí mismo en relación con los demás: una identidad. (p.14)

En este sentido, es preciso indicar que la escuela busca ser un espacio posibilitador de estas características, donde los profesores se comprometan con sus estudiantes en la promoción del respeto y la 
autonomía, donde el estudiante sea un componente activo y participativo de la sociedad "construir la ciudadanía en la escuela es establecer un escenario de vivencia del otro, valorando la diversidad de vivencias culturales, potenciando la asociatividad y buscando romper con las desigualdades instaladas". (Redón, S. y Toledo, L., 2009, p.15)

La escuela, en tanto espacio público, tiene el deber de promover una cultura escolar hermanada con el diálogo comunitario, la convivencia y la defensa de la alteridad. Los docentes tienen el rol protagónico en esta tarea y en la activación del diálogo intergeneracional que esto requiere. Desde esta perspectiva, sería el docente quien estaría particularmente involucrado en el rescate de la ciudadanía, siendo entonces el encargado de trabajar la formación ciudadana.

La formación ciudadana en el currículum nacional se podría definir en tres aspectos:

- La institucionalidad política se amplia como aprendizaje, integrando temáticas y problemáticas actuales de la sociedad.

- La formación ciudadana se presenta como un componente que se manifiesta en varios niveles y disciplinas, abordando totalmente la secuencia escolar.

- La formación ciudadana se amplía a la adquisición de saberes, procedimientos y actitudes propias del aprendizaje en democracia.

Al considerar estos lineamientos que se encuentran en el Marco Curricular (2009), es posible identificar que el debate, la deliberación, la investigación, la conversación sobre las trayectorias de la humanidad en su contexto espacial y temporal, así como la empatía con actores sociales diferentes, forman parte de los principios fundamentales que el curriculum quiere aportar, que es dialogante y se enriquece con una formación ciudadana, que hoy es posible de ser abordada transversalmente en los distintos sectores de aprendizaje.

Sin embargo, es importante no olvidar que las tensiones persisten debido a que el lugar que ocupa el docente en la actualización curricular, responde al rol de implementador de modificaciones preescritas, sin ser parte de su diseño, ni ser considerado como "(...) el actor curricular principal es el educador, interactuando con el educando y su mundo vital (...)" (Pinto,2007, p.116). Al considerar al docente como implementador, se genera una problemática respecto a la comprensión de los ajustes, pues, a pesar de ser considerados una actualización existen discusiones que se tienen que plantear respecto al diseño y estas no se generan por sí solas, sino responden a un proceso de reflexión y discusión más profunda referido a lo que se va a implementar, en esta lógica de incertidumbre docente es que el curso se presenta como un espacio de participación social como lo propone Escudero (1988) en que el docente a pesar de ser considerado como implementador, logró encontrar posibilidades de acción referidas a la reinterpretación del curriculum a partir de una lectura apropiada y de su relación con prácticas educativas.

En este sentido, el curso Formación para la democracia y el ejercicio de los derechos, fue concebido como un aporte que intentó disminuir aquella tensión, ampliando la función del docente, desde simple implementador de un saber preescrito y normado a un docente crítico, reflexivo y dinamizador del contenido. 


\section{GRANDES METAS: LOS ALTIBAJOS DE UN CURSO EN EJECUCIÓN}

El curso fue diseñado en formato b-learning considerando la importancia de las TIC's en el ámbito educativo, por lo cual contó con un aula virtual que contenía los módulos, herramientas y actividades a ser desarrolladas durante los tres meses de duración. Asimismo, fueron programados tres encuentros presenciales los cuales fueron estratégicamente posicionados, uno al inicio, otro durante el desarrollo y por último, uno como cierre del curso.

Los módulos que componían el curso eran cuatro, siendo el primero un módulo introductorio a los aspectos tecnológicos del curso, mientras que los siguientes tres módulos se centraron en tres grandes ejes: Formación Ciudadana, Organización Política y Derechos Ciudadanos. Denominándose cada módulo de la siguiente forma:

- Módulo 1: Claves para abordar la formación ciudadana

- Módulo 2: Claves para abordar la organización política en democracia

- Módulo 3: Claves para abordar los derechos ciudadanos y los derechos humanos: sus componentes, historicidad y vínculos en común.

Cada módulo poseía actividades de inicio, desarrollo y cierre, siendo las actividades denominadas Reflexiones y Aplicaciones de carácter obligatorias y calificadas. Además se establecieron dos test $^{23}$, el primero al iniciar el curso y el segundo al finalizarlo, siendo estos una de las herramientas más clarificadoras y reveladoras con respecto a las posibilidades y ventajas que este curso otorgó a sus participantes. Lo anterior se dio, porque ambas herramientas fueron construidas en formato espejo, entregando respuestas de un pre-test, o sea, las expectativas antes de realizar el curso, y un pos-test, es decir, el cumplimiento o no de aquellas expectativas antes mencionadas.

En este sentido, fue posible establecer que los beneficios que los participantes del curso deseaban obtener fueron cumplidos, ellos en su gran mayoría identificaron un mejoramiento del quehacer de aula, esto se encuentra asociado a planos de actualización curricular en ámbitos de didáctica; equivaliendo a una reflexión amplia sobre los planos que comprenden la Formación Ciudadana asociado al aula, al mismo tiempo, ampliaron su comprensión y manejo de recursos curriculares tales como el mapa de progreso "Democracia y Desarrollo", que es parte de las innovaciones curriculares que vienen de la mano con la actualización curricular.

A su vez, es importante destacar la visión integradora que comienza a desarrollar el docente participante, pues va paulatinamente desplazando su discurso efectivista con respecto a la configuración del ciudadano como el simple sujeto que da su voto o que es un buen patriota, se volviéndose mucho más importantes la participación ciudadana como eje. Claramente, existe un porcentaje bajo de profesores que mantienen una visión tradicional de la ciudadanía, relacionándose mucho más con lo que se conocía como educación cívica en el curriculum sin ajustes.

Por otro lado, los docentes participantes reconocen que la formación ciudadana está dirigida a los estudiantes constituidos como individuos, son estos agentes particulares los cuales deben co-

23 Los test con sus respectivos gráficos y análisis se encuentran disponibles en el informe final del curso, material exclusivo de PIIT-UAHC y CPEIPVirtual.. 
nocer tanto derechos como deberes asociados a la vida en democracia. Para los docentes, existe la necesidad de generar valoración de las instancias de participación democrática, esto se traduce en el manejo de conocimientos teóricos sobre ciudadanía, así como socio-históricos de los mismos, lo anterior propone formas de comprender los contenidos asociados a la formación ciudadana.

De esta manera, los docentes participantes proponen la formación ciudadana como una instancia de adquisición de conocimientos tanto conceptuales (en ámbitos teóricos, y también históricos de la constitución de derechos por ejemplo, entre otros), también procedimentales (asociados a las instancias diseñadas para participación democrática) y además actitudinales (en planos de valoración de la convivencia democrática, el pluralismo, de los derechos, y por cierto de los deberes).

Así mismo, los docentes participantes reconocen inicialmente la noción de colectivo, los estudiantes se encuentran asociados a un colectivo social, con construcciones espaciales, culturales, económicas, entre otras. Esto indicaría inicialmente, que la formación ciudadana representa un manejo conceptual (como lo apuntado en la categorización anterior), sin embargo esto además propone una responsabilidad esencial con la valoración de una sociedad igualitaria y justa, en el amplio sentido de la palabra.

De esta manera, los docentes participantes relevan la generación de diversas instancias de participación democrática, en donde los estudiantes movilicen los conocimientos adquiridos en su formación, apuntando a un visión compleja y crítica de la realidad que habitan, apuntando a la búsqueda de resoluciones de conflictos sociales de manera, democrática y pluralista.

Es así como es posible detectar un amplio porcentaje de particicipantes que declaron finalmente que la formación ciudadana, posee relación con la forma de desarrollar habilidades y competencias en los estudiantes para la resolución de conflictos, ingresando un contenido procedimental y actitudinal, no sólo conceptual. Apartándose de sus primeras declaraciones que solo consideraban la promoción del conocimiento de los derechos y saberes de que cada ciudadano.

\section{FINALMENTE: LOS RESULTADOS FUERON IMPORTANTES, PERO LOS CONFLICTOS SE MANTIENEN}

El curso Formación para la democracia y el ejercicio de los derechos claramente representó un espacio de posibilidad para el accionar curricular del profesorado, pues, entregó herramientas que proporcionaron autonomía para que el docente participante pudiese realizar una lectura consiente del dispositivo curricular y pensar desde este en su propuesta didáctica. Sin embargo el curso, representó un nicho de actualización curricular en un escenario lleno de incertidumbre para los actores educativos, pues los ajustes curriculares 2009 se plantean al profesorado como una actualización que debe ser leída de manera automática por quienes la implementan, sin dar lugar a los espacios de reflexión y discusión, ni considerar los aportes que podrían surgir hacia el curriculum nacional, desde la experiencias educativas.

Reiterando uno de los aportes del curso, es posible asumir que frente a una actualización es necesario promover prácticas de perfeccionamiento docentes que promuevan una apropiación curricular. Por lo tanto, esta instancia consideró que la posibilidad de ejercer autonomía va de la mano con la necesidad de mayor apropiación de nuevas teorías e investigaciones, y esto porque, el docente como profesional debe estar a la vanguardia del conocimiento, sin esta posibilidad es difícil que 
logre su autonomía profesional y se pueda despojar de la prescripción como una instrucción totalitaria a implementar.

De modo que, pensando en el escenario en el que finalizó el curso, esto no sólo permite realizar una reflexión respecto a los aportes de su ejecución, sino que permite pensar también en los nuevos desafíos que el curriculum chileno presupone y en problemáticas que deben enfrentar los profesores, y por ende los estudiantes. Es evidente que los conflictos entre la autonomía del docente, la participación social (participación ciudadana) y la prescripción curricular se mantienen, quizás uno de los mayores aportes que otorgó este curso, fue justamente el cuestionamiento del profesional de la educación sobre su quehacer docente y su efectiva injerencia en la toma de decisiones en cuanto a educación se refiere.

\section{BIBLIOGRAFÍA}

Colegio de profesores, Magisterio junto a premios nacionales desaprueban ajustes curriculares, $<$ http:// www.colegiodeprofesores.cl/index2.php?option $=$ com_content\&task=emailform\&id $=547 \&$ it emid=2> consultado el 9 de diciembre de 2010 - Recuperado el 30 de noviembre de 2011

Escudero, J. (1988). La innovación y la organización escolar. En Pascual, R. (Coord.). La gestión educativa ante la innovación y el cambio. Editorial Narcea, Madrid.

Gysling, J. (2003). Reforma curricular: Itinerario de una transformación cultural. En C.Cox (Ed.) Politicas educacionales en el cambio de siglo: Reforma educacional Chilena. Editorial Universitaria, Santiago.

Gimeno Sacristán, J. (2001). La ciudadanía como metáfora para la educación. Revista Docencia, (15), [p. 12-21].

Ministerio de Educación (2009). Objetivos Fundamentales y contenidos Minimos Obligatorios de la Educación Básica y Media Actualización 2009. Gobierno de Chile, Santiago.

Pinto R. (2007). El currículo crítico. Una pedagogía transformativa para la educación latinoamericana. Pontificia Universidad Católica de Chile. Santiago, Chile.

Redón, S. y Toledo, L. (2009). Escuela, democracia y ciudadanía. Revista Docencia, (39), [p. 12-17].

Artículo recibido 20 - 09 - 11. Aprobado 25 - $11-11$. 